\title{
Fasudil Protects Against the Myocardial Hypertrophy of Transverse Aortic Constriction Via Modulating the Expression of Caspase-3 and Bcl-2 Protein \\ Le LI ${ }^{a},{ }^{\star}$, Hao SHANG ${ }^{a}$, Cai-ling ZHANG ${ }^{b}$, Hou-quan TAO $^{c}$ and Jir- kun $\mathrm{CHENG}^{\mathrm{a}}$ \\ aSchool of Pharmacy, Zhejiang University of Technology, Hangzhou, Zhejiang 310014,PR China

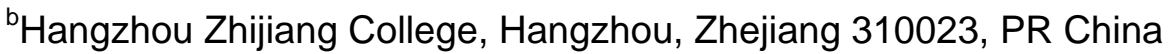 \\ ${ }^{c}$ Center Lab of Zhejiang Province People Hospital, Hangzhou, Zhejiang 310014, PR China \\ E-mail:lile_1856@163.com
}

Keywords: Fasudil, Expression of Caspase-3 and Bcl-2 Protein, Cardiac Hypertrophy, Transverse, Aortic Constriction.

\begin{abstract}
Objectives: To investigate the effects of fasudil (Fas) on myocardial hypertrophy in rats. Methods Rats were randomly divided into 5 groups: control, transverse aortic constriction (TAC) model, Fas $(5 \mathrm{mg} / \mathrm{kg})$ plus TAC, TAC plus Fas $(20 \mathrm{mg} / \mathrm{kg}$ ), and TAC plus captopril (Cap, positive drug). After administering treatments for eight weeks, myocardial intracellular $\mathrm{Ca}^{2+}$ ion, cardiac hemodynamic parameters and histological changes, as well as NO and SOD levels of serum were measured. Protein expression changes in Caspase- 3 and Bcl-2 were detected. Results Compared with the control group, rats in the TAC group showed increases in cardiac and left ventricular weight indexes, and had larger myocardial fiber diameters, as well as an increased incidence of myocardial fibrosis. Treatment of TAC-induced rats with Fas and Cap reduced the degree of these changes, improving myocardial contraction and relaxation functions, and significantly $(p<0.05)$ reducing the free $\mathrm{Ca}^{2+}$ ion concentration of myocytes. The results indicated that Fas or Cap treatment can alleviate myocytes apoptosis. Furthermore, increasing expression of Caspase- 3 and decreasing expression of $\mathrm{Bcl}-2$ were observed in the TAC model group, and these changes could be alleviated by treatment with Fas and Cap. Conclusions Fas treatment significantly improved cardiac hypertrophy, which might be related to modulating the expression of Caspase- 3 and Bcl-2 protein, enhancement of NO release of myocytes in rats after TAC
\end{abstract}

\section{Introduction}

Cardiac hypertrophy $(\mathrm{CH})$ is an adaptive response to a variety of pathological cardiac myocyte stimulations. In an emergency situation, lasting pathological cardiac hypertrophy is accompanied by interstitial fibrosis, changes in gene expression, systolic dysfunction, abnormal energy metabolism, and electrophysiological characteristics, which eventually lead to decompensated heart failure and serious harm to human health [1, 2], $\mathrm{CH}$ has been listed as an independent risk factor in significantly higher incidences of mortality by cardiovascular disease [3]. Clinical studies have shown that many cardiovascular diseases, such as hypertension, coronary atherosclerosis, valvular disease, myocardial infarction, and hypertrophic cardiomyopathy are associated with cardiac hypertrophy. Many intrinsic and extrinsic 
stimuli, such as endocrine disorders, ischemia, neurohormonal factors, and cytokines can induce cardiac hypertrophy, which is characterized by myocyte disarray and interstitial fibrosis.

In recent years, a considerable number of studies have shown that apoptosis plays an important role in many cardiovascular diseases, such as hypertension, cardiac hypertrophy, heart failure, and atherosclerosis [4].Generally, pressure overloadinduced cardiac myocyte apoptosis is an important factor in myocardial hypertrophy. Cardiomyocyte apoptosis can be caused by high blood pressure, mechanical force generated by the renin-angiotensin system, free radicals, ischemia, hypoxia, and cell damage $[5,6]$. Genes involved in the regulation of myocardial apoptosis include bcl2/bax, c-myc and p53.Overload factors induce apoptosis of the myocardial intracellular pathway mainly through the mitogen-activated protein kinase(MAPK) pathway. Specifically, these stimuli activate the mitogen-activated protein kinase kinase (MKK3) and p38MAPK pathways [7, 8].

Fas is clinically used to treat a variety of causes of ischemic cerebrovascular disease, and is the most commonly used clinical Rho associated coiled-coil forming protein kinase (ROCK) inhibitor [9]. Fas has been reported to inhibit hypertension and angiotensin II (AngII) induced cardiac hypertrophy, as well as the chronic congestive heart failure caused by hypertension, but the specific mechanisms of Fas action need further study [10]. Specifically, the effects of Fas on cardiac hypertrophy and cardiac myocyte apoptosis in rats with TAC have been less reported. In this study, a TACinduced cardiac hypertrophy model in rats was utilized to observe the effects of Fas on cardiac hypertrophy and myocardial apoptosis..

\section{Material and Methods}

\section{Materials}

Fas hydrochloride was purchased from red Tianjin Pharmaceutical Co., Ltd., batch 1301241 and used for injection at a concentration of $15 \mathrm{mg} / \mathrm{ml}$. Captopril(Cap) was obtained from Changzhou Pharmaceutical Co., Ltd., batch number 1302131. Sodium pentobarbital and fura-2/AM were purchased from Sigma. AngII, endothelin (ET), and the atrial natriuretic peptide (ANP) radioimmunoassay kit were purchased from Beijing Furui biological Engineering. The nitric oxide (NO) and the superoxide dismutase (SOD) kit were purchased from Nanjing biotechnology companies. The TUNEL kit, anti-digoxin antibody, and Caspase-3 and Bcl-2 antibodies were purchased from Wuhan Boster biological Engineering Co., Ltd. Other reagents were of analytical grade. The BL-420E-type four polygraph was obtained from Chengdu Science and Technology Co., Ltd. The BMJ-type embedding machine was purchased from Changzhou Electronic Instrument Factory. The slicer was purchased from LEICA2135. The speeding cold centrifuge was purchased from Beckman. The FJ 2008PS autoimmune count analyzer was obtained from the Xi'an nuclear Instrument Factory. The 722 spectrophotometer was purchased from the Shanghai third Analytical Instrument Factory. The automated tissue processor was obtained from Leica. Microscopy and imaging systems were purchased from Nikon. The cryogenic freezer $\left(-20^{\circ} \mathrm{C}\right)$ was obtained from Siemens. The transfer electrophoresis tank was obtained from Beijing sixty-one Instrument. The gel image processing system was purchased from Bio-Rad. 


\section{Animal Model and Group Administration}

Fifty-four healthy adult male Wistar rats ,aged 8-10 weeks and weighing $258.54 \pm 19.58 \mathrm{~g}$, were purchased from the West Coast Poole-Rubicam animal company. Rats were housed at $\left(25 \pm 2^{\circ} \mathrm{C}\right)$ with $55 \%-60 \%$ humidity and daytime fluorescent illumination occurring from 8:00 to 17:00. No more than 44 rats were randomly divided into four groups (in addition to a sham group with 10 rats, which were chosen using a random table method). These groups included the abdominal aortic coarctation model group(TAC model group), a TAC group receiving Fas $(5 \mathrm{mg} / \mathrm{kg})$ treatment, a TAC group receiving $20 \mathrm{mg} / \mathrm{kg}$ of Fas treatment, and a TAC group receiving Cap $(50 \mathrm{mg} / \mathrm{kg}$, positive control group). The pressure overload cardiac hypertrophy model was prepared by the TAC surgery. Rats were fed for 5 days and then fasted for 12 hours. After fasting, rats were anesthetized with $0.5 \%$ sodium pentobarbital, delivered intraperitoneally, at a concentration of $45 \mathrm{mg} / \mathrm{kg}$. After anesthetization, the abdomen was opened along the ventral white line, and the abdominal aorta was isolated. The renal arteries were clipped (up to $0.5 \mathrm{~cm}$ ) with a silver clip of $0.70 \mathrm{~mm}$ diameter. This ensured that the abdominal aortic artery was constricted to $60 \%-70 \%$. Following confirmation that there was no bleeding, the organ was reset and the abdomen was closed and a model of pressure overload cardiac hypertrophy was set up. The operation performed in the sham group rats was similar to those mentioned above, but without TAC. After TAC, the Fas groups were administered intraperitoneally once per day with either 5 or $20 \mathrm{mg} / \mathrm{kg}$ of the compound. The Cap group was given $50 \mathrm{mg} / \mathrm{kg}$ of the compound by intra-gastric delivery once per day. The control group was given an equal volume of sterile water by intraperitoneal injection once a day for 8 weeks.

\section{Hemodynamics and Cardiac Mass Index, Left Ventricular Mass Index Detection}

After 24 hours with no drug treatment, rats of each group were anesthetized with $0.5 \%$ sodium pentobarbital at $45 \mathrm{mg} / \mathrm{kg}$, delivered intraperitoneally. The right carotid artery was isolated, and the distal end of the catheter was ligated. The other end of the conduct was connected with the baroreceptor in the physiological recorder, and cut like a horseshoe. The artery was cut with a small hole, which was filled with heparin saline. A 1-mm diameter polyethylene catheter from the right carotid artery was slowly inserted into the proximal end of the push. The electric physiological pressure instrument displayed a wave pattern on the left ventricular pressure graph, indicating that catheter has been inserted the left ventricle. After 15 minutes of stability, the heart rate (HR), mean blood pressure (MAP), left ventricular systolic pressure (LVSP), left ventricular end diastolic pressure (LVEDP), and the changes in the left ventricular pressure the maximum rate $( \pm \mathrm{dP} / \mathrm{dtmax})$ were measured.

After hemodynamic testing, the rats were sacrificed. The weights of rats (body weight, BW) were determined using an electronic balance. The heart was rapidly removed through thoracotomy, and residual blood was washed with phosphate buffered saline (PBS), and then the residual liquid was dried with filter paper. The full heart weight (HW) was determined using a precision electronic balance, and then the heart was placed on ice. The great vessels, pericardium tissues, and the atrial and the right ventricle were removed along the ventricular septal. The left ventricle and interventricular septum were retained and the left ventricular mass (LVM) was weighed. The cardiac mass index (HMI) was calculated according to the following formula: $\mathrm{HMI}=$ heart weight/body weight $(\mathrm{HW} / \mathrm{BW})$, left ventricular mass index (LVMI), LVMI=left ventricular weight/body weight (LVW/BW). 


\section{Blood Biochemical Parameters of Rats}

Before hemodynamic parameters were measured, blood were took from each group of rats from the femoral artery, to prepare serum. Serum levels of AngII, ET, ANP, NO, and SOD were detected according to the relevant kit instructions.

\section{Cardiac Pathology}

After hemodynamics testing, the rats were sacrificed. Left ventricular muscle tissues were fixed in $4 \%$ paraformaldehyde. Following fixation, tissues were embedded in paraffin and stained by H\&E and MASSON. Stained tissues were analyzed using high-resolution color with the HM IAS22000 medical image analysis system. MASSON staining on each mouse were randomly selected 6 vision, measurement of myocardial fiber diameter (CMD), averaged.

\section{Determination of Myocardial Intracellular Free Calcium Concentration}

After hemodynamic testing, the rats were sacrificed. Ventricular myocytes were isolated and cardiac load Fura-2 cells were placed under a fluorescence microscope. Calcium fluorescence was determined by excitation with a wavelength of 340/380 nm and an emission wavelength of $510 \mathrm{~nm}$. Data was analyzed using Felix special fluorescent signal processing software.

\section{TUNEL Assay of Myocardial Apoptosis}

Rats were sacrificed and heart tissues were removed and fixed in $4 \%$ neutral formalin. Following graded alcohol dehydration, the middle of the left ventricle was embedded into paraffin and sectioned $(5 \mu \mathrm{m})$. Sections were soaked in alcohol gradients, washed with PBS, soaked in fresh $3 \% \mathrm{H} 202$ for $10 \mathrm{~min}$, and digested with proteinase $\mathrm{K}$ at $37^{\circ} \mathrm{C}$ for 15 minutes. Following digestion, samples were washed with PBS and then incubated with a sliced TdT and DIG-d-UTP mixture. Samples were placed in a moist chamber at $37^{\circ} \mathrm{C}$ for 2 hours with a blocking solution. Samples were then treated with a biotinylated anti-digoxin antibody (DAB color) and slightly stained by hematoxylin. Using the TUNEL staining kit, myocardial cells positive for apoptosis contained brown granules. These cells were counted under $\times 400$ objective observation in 10 nonoverlapping fields of view.

\section{Western Blot of Caspase-3 and Bcl-2 Protein Levels.}

Western blotting for Caspase-3 and Bcl-2 protein levels was carried out as previously reported[11]. but is summarized briefly as follows: Samples were mixed with protein loading buffer, boiled, and separated by $10 \%$ SDS-PAGE electrophoresis. Proteins were transferred using semi-dry transfer film. Primary antibodies were incubated overnight. After secondary antibody incubation, samples were treated with ECL and developed using chemiluminescence film exposure in the darkroom.

\section{Statistical Analysis}

Statistical analysis was performed using SPSS17.0 software. Measurement data was expressed as mean \pm standard deviation. Groups were compared using ANOVA (OneWay ANOVA), and pair-wise comparisons were made using the LSD method. Statistically significant differences required a p-value $<0.05$. 


\section{Results}

\section{Physiological State of Rats}

A total of forty-six rats survived to completion of the experiment, while 8 rats died. Four of these rats were in the TAC model group, one was in the Cap group, two were in the low-dose Fas-treatment group, and one was in the high-dose Fas-treatment group. All rats in the control group survived. The main cause of death was heart failure. During the first week after surgery, four groups of animals showed reduced activity, deteriorating hygiene, and decreased food and water intake. These habits improved after two weeks. Throughout the experiment, sham rats remained healthy, as exhibited by shiny coats, sensitive actions, and a high level of responsiveness to the surrounding environment. In contrast, rats in the late TAC model group exhibited dull coats and were apathetic and slow. The results showed that conditions were better for TAC rats treated with Fas or Cap.

\section{Fas Impact on Heart Weight Index, Left Ventricular Mass Index and Myocardial Fiber Diameter}

Compared with the TAC model group, the Fas- and Cap-treated groups had statistically significantly lower HMI, LVMI and CMD levels (Table 1).

Table 1. Comparison of muscle fiber diameter (CMD), left ventricular myocardial mass index (LVMI) and heart weight index (HWI) in each group of rats $(\mathrm{x} \pm \mathrm{S})$

\begin{tabular}{llllll}
\hline Group & $n$ & dose $(m g / K g)$ & $H W I(m g / g)$ & $L V M I(m g / g)$ & $C M D(\mu M)$ \\
\hline Sham & 10 & - & $2.63 \pm 0.13$ & $1.93 \pm 0.11$ & $12.57 \pm 0.29$ \\
TAC model & 7 & - & $3.23 \pm 0.25^{\mathrm{a}}$ & $2.65 \pm 0.21^{\mathrm{a}}$ & $17.7 \pm 0.16^{\mathrm{a}}$ \\
Fas & 9 & 5 & $3.07 \pm 0.15^{\mathrm{b}}$ & $2.31 \pm 0.12^{\mathrm{b}}$ & $14.42 \pm 0.37^{\mathrm{b}}$ \\
Fas & 10 & 20 & $2.89 \pm 0.22^{\mathrm{c}}$ & $2.19 \pm 0.23^{\mathrm{c}}$ & $12.37 \pm 0.27^{\mathrm{c}}$ \\
Cap & 10 & 50 & $2.97 \pm 0.24^{\mathrm{b}}$ & $2.25 \pm 0.23^{\mathrm{c}}$ & $13.63 \pm 0.24^{\mathrm{c}}$ \\
\hline
\end{tabular}

Note: Compared with the Sham group, ${ }^{\mathrm{a}} \mathrm{P}<0.05$; Compared with TAC model group, ${ }^{\mathrm{b}} \mathrm{P}<0.05,{ }^{\mathrm{c}} \mathrm{P}<0.01$ )

\section{Hemodynamics}

Results from hemodynamics analysis (Table 2) showed that, compared with the control group, rats in the TAC model group had significantly $(\mathrm{P}<0.05)$ higher levels of MAP, LVSP, LVEDP and $\pm \mathrm{dP} / \mathrm{dtmax}$. These levels dropped significantly after treatment with a high dose of Fas or with Cap.

Table 2. The changes of cardiac hemodynamic parameters in each group of rats after eight weeks $(\mathrm{x} \pm \mathrm{S})$

\begin{tabular}{|c|c|c|c|c|c|c|c|c|}
\hline Group & $n$ & $\begin{array}{l}\text { dose } \\
m g / k \\
g\end{array}$ & $\begin{array}{l}\text { HR } \\
\text { beat/min }\end{array}$ & $M A P k P a$ & $L V S P k P a$ & $\begin{array}{l}L V E D P \\
k P a\end{array}$ & $\begin{array}{l}+d P / d t_{\max } \\
\mathrm{kPa} / \mathrm{s}\end{array}$ & $\begin{array}{l}-d P / d t_{\max } \\
\mathrm{kPa} / \mathrm{s}\end{array}$ \\
\hline Sham & 10 & - & $348 \pm 21$ & $\begin{array}{l}16.24 \pm 1.6 \\
2\end{array}$ & $10.33 \pm 1.92$ & $0.53 . \pm 0.15$ & $286 \pm 42$ & $209 \pm 41$ \\
\hline $\begin{array}{l}\text { TAC } \\
\text { model }\end{array}$ & 7 & - & $329 \pm 19$ & $\begin{array}{l}20.38 \pm 1.8 \\
9^{\mathrm{a}}\end{array}$ & $14.81 \pm 2.34$ & $0.95 \pm 0.13$ & $589 \pm 111^{\mathrm{a}}$ & $369 \pm 89^{\mathrm{a}}$ \\
\hline Fas & 9 & 5 & $345 \pm 14$ & $\begin{array}{l}21.02 \pm 2.1 \\
4^{\mathrm{a}}\end{array}$ & $12.16 \pm 1.78$ & $0.79 \pm 0.16$ & $482 \pm 93^{b}$ & $324 \pm 69$ \\
\hline Fas & 10 & 20 & $346 \pm 15$ & $\begin{array}{l}20.74 \pm 2.0 \\
2^{\mathrm{a}}\end{array}$ & ${ }_{C}^{10.12 \pm 1.47}$ & $0.69 \pm 0.14^{\mathrm{b}}$ & $401 \pm 82^{C}$ & $269 \pm 73^{b}$ \\
\hline Cap & 10 & 50 & $348 \pm 16$ & $\begin{array}{l}20.85 \pm 2.0 \\
5^{\mathrm{a}}\end{array}$ & ${ }_{C}^{11.09 \pm 1.51}$ & $071 \pm 0.14^{\mathrm{C}}$ & $399 \pm 81^{\mathrm{C}}$ & $254 \pm 60^{C}$ \\
\hline
\end{tabular}

Note: Compared with the Sham group, ${ }^{a} \mathrm{P}<0.05$; Compared with TAC model group, ${ }^{\mathrm{b}} \mathrm{P}<0.05,{ }^{\mathrm{c}} \mathrm{P}<$ $0.01)$. 


\section{Assay of Blood Biochemical Parameters}

Compared to the control group, significantly higher levels of AngII, ET, and ANP, and significantly lower levels of NO and SOD in the serum of rats were observed in the TAC model group. These changes were alleviated following treatment with Cap and Fas (Table 3).

Table 3 Comparison of the blood biochemical indicators in each group of rats $(x \pm S)$

\begin{tabular}{|c|c|c|c|c|c|c|c|}
\hline $\begin{array}{l}\text { Grou } \\
p\end{array}$ & $n$ & $\begin{array}{l}\text { Dose } \\
\mathrm{mg} / \mathrm{kg}\end{array}$ & Ang// $p g / m l$ & $E T p g / m l$ & $A N P p g / m l$ & $\mathrm{NO} \mathrm{U/ml}$ & $S O D U / m l$ \\
\hline Sham & 10 & - & $69.2 \pm 14.24$ & $\begin{array}{l}116.4 \pm 11 . .3 \\
2\end{array}$ & $\begin{array}{l}418.25 \pm 22.2 \\
1\end{array}$ & $\begin{array}{l}279.36 \pm 40.1 \\
2\end{array}$ & $\begin{array}{l}52.16 \pm 5.2 \\
3\end{array}$ \\
\hline $\begin{array}{l}\text { TAC } \\
\text { mode } \\
1\end{array}$ & 7 & - & $\begin{array}{l}144.16 \pm 15.1 \\
3^{\text {a }}\end{array}$ & $\begin{array}{l}172.0 \pm 15.4 \\
6^{\mathrm{a}}\end{array}$ & $\begin{array}{l}631.1 \pm 46.22 \\
\mathrm{a}\end{array}$ & $\begin{array}{l}117.22 \pm 27.8 \\
2^{\mathrm{a}}\end{array}$ & $\begin{array}{l}39.13 \pm 3.2 \\
1^{\mathrm{a}}\end{array}$ \\
\hline Fas & 9 & 5 & $\begin{array}{l}101.12 \pm 17.2 \\
2^{\mathrm{b}}\end{array}$ & $\begin{array}{l}146.3 \pm 14.1 \\
4^{\mathrm{b}}\end{array}$ & $\begin{array}{l}514.64 \pm 48.4 \\
6^{b}\end{array}$ & $\begin{array}{l}173.34 \pm 40.2 \\
3^{c}\end{array}$ & $\begin{array}{l}48.24 \pm 2.5 \\
6^{c}\end{array}$ \\
\hline Fas & 10 & 20 & $81.31 \pm 10.14^{\mathrm{c}}$ & $\begin{array}{l}109.4 \pm 10.6 \\
3^{\mathrm{c}}\end{array}$ & $461.7 \pm 39.24^{c}$ & $\begin{array}{l}204.24 \pm 48.2 \\
7^{\mathrm{c}}\end{array}$ & $\begin{array}{l}59.25 \pm 3.5 \\
4^{c}\end{array}$ \\
\hline Cap & 10 & 50 & $78.27 \pm 7.42^{c}$ & $\begin{array}{l}111.6 \pm 12.7 \\
5^{c}\end{array}$ & $\begin{array}{l}453.14 \pm 38.3 \\
2^{c}\end{array}$ & $\begin{array}{l}211.14 \pm 37.8 \\
4^{c}\end{array}$ & $\begin{array}{l}51.87 \pm 2.9 \\
9^{c}\end{array}$ \\
\hline
\end{tabular}

Note: Compared with the Sham group, ${ }^{\mathrm{a}} \mathrm{P}<0.05$; Compared with TAC model group, ${ }^{\mathrm{b}} \mathrm{P}<0.05,{ }^{\mathrm{c}}$ $\mathrm{P}<0.01)$.

\section{Cardiac Pathology}

As displayed in Figure 1A, 1B, H\&E staining combined with Masson staining revealed normal-sized cells in the control group. The control group also displayed neatly arranged muscle fibers. In contrast, myocardial cells from the TAC model group were disordered, with muscle fibers that were thickened with hypertrophy, and containing gaps, indicating edema. As these gaps widened, the infiltration of inflammatory cells in the tissues was observed. Additionally, in the TAC group, the obvious fibrosis of perivascular and interstitial tissue was observed. Compared with the pathological changes, the Fas and Cap groups displayed improved myocardial fibers in the TAC model group, as well as myocardial cells arranged neatly, with a narrowed gap, and little tissue infiltration of inflammatory cells. Furthermore, the reduced fibrosis of perivascular and interstitial tissues in the Fas and Cap treatment groups were observed . 

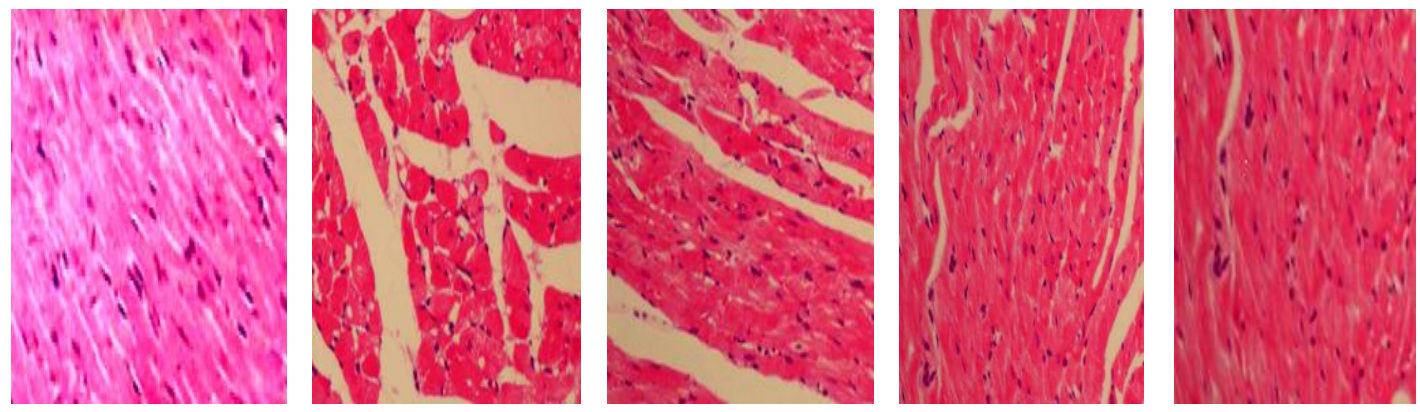

\section{A: sham B: TAC Model C: Model+5mg/kgFa D: Model+20mg/kg Fas E:Model+50mg/kg Cap}

Figure 1 A Myocardial pathology observations in each group of rats (HE staining, 200, $x \pm S, n=7-10$ ), Bars represent $50 \mu \mathrm{m}$

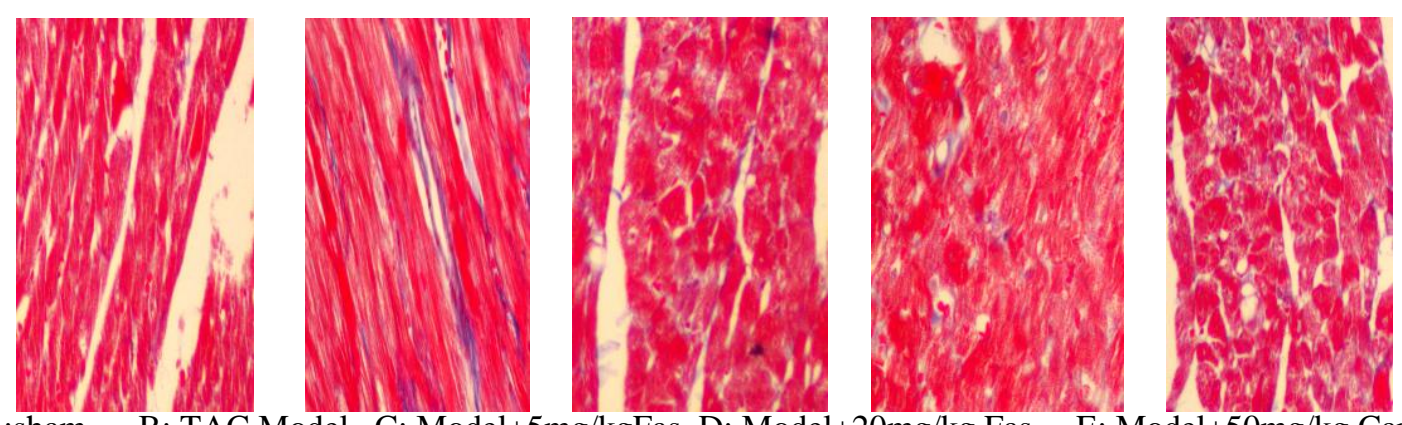

A:sham B: TAC Model C: Model+5mg/kgFas D: Model $+20 \mathrm{mg} / \mathrm{kg}$ Fas $\quad$ E: Model $+50 \mathrm{mg} / \mathrm{kg} \mathrm{Cap}$

Figure 1 B. Myocardial pathology observations in each group of rats (Masson staining, 200, Blue means

fibrosis, $\mathrm{x} \pm \mathrm{S}, \mathrm{n}=7-10$ )

\section{Changes in Intracellular Free $\mathrm{Ca}^{2+}$ Ion Concentration in Myocardial Cells}

The results showed that the intracellular concentrations of free $\mathrm{Ca}^{2+}$ ions in cells from the TAC model group were significantly higher than those of cells in the control group (Table 4). Compared with the TAC model group, the concentrations of free $\mathrm{Ca}^{2}$ ${ }^{+}$ions in myocardial cells in the Fas and Cap groups were significantly $(\mathrm{P}<0.05)$ lower. Furthermore, the $\mathrm{Ca}^{2+}$ ion concentrations in myocardial cells in the Fas high-dose group were not statistically different from the control group. While the free $\mathrm{Ca}^{2}{ }^{+}$ion concentrations in myocardial cells in the Cap-treatment group were significantly higher than those in the control group, they were lower than that of the TAC model group. These data show that treatment with a high dose of Fas reduces $\mathrm{Ca}^{2}+$ ion concentrations in myocardial cells. Additionally, the results indicated that Fas treatment has a more pronounced effect on myocardial free $\mathrm{Ca}^{2+}$ concentrations than Cap does.

Table 4 Intracellular calcium changes of myocardial cells in each group of rats after 8 weeks $(x \pm \mathrm{S})$

\begin{tabular}{llll}
\hline Group & $n$ & dose $(\mathrm{mg} / \mathrm{kg})$ & {$\left[\mathrm{Ca}^{2+}\right] i$} \\
\hline Sham & 10 & - & $97.84 \pm 7.43$ \\
TAC Model & 7 & - & $163.36 \pm 15.13^{\mathrm{a}}$ \\
Fas & 9 & 5 & $139.35 \pm 11.26^{\mathrm{ab}}$ \\
Fas & 10 & 20 & $113.31 \pm 11.02^{\mathrm{c}}$ \\
Cap & 10 & 50 & $122.15 \pm 10.17^{\mathrm{ac}}$ \\
\hline
\end{tabular}

Note: Compared with the Sham group, ${ }^{\mathrm{a}} \mathrm{P}<0.05$; Compared with TAC model group, $\left.{ }^{\mathrm{b}} \mathrm{P}<0.05,{ }^{\mathrm{c}} \mathrm{P}<0.01\right)$. 


\section{TUNEL Assay of Myocardial Apoptosis}

Following staining of myocardial tissues with 3,3 - diaminobenzidine (DAB) and hemotoxylin, normal nuclei should appear blue, and of a normal size, while apoptotic nuclei should stain with a brown/yellow color. In our analysis, the results showed that the control group contained very few apoptotic cells. In contrast, in the TAC model group, frequent incidences of myocardial apoptosis were observed. The apoptotic index (AI) of the TAC group was significantly higher than that of the control group $(\mathrm{P}<0.05)$ (Table 5). Additionally, the results showed that treatment with Fas or Cap significantly $(\mathrm{p}<0.05)$ reduced the incidence of apoptotic cells following TACinduction, however, these cells were still observed more frequently than in the control group (Fig. 3, Table 5).

Table 5 The changes of myocardial cell apoptosis in each groups of rats after eight weeks $(\mathrm{x} \pm \mathrm{S})$

\begin{tabular}{llll}
\hline Group & $n$ & $\operatorname{dose}(\mathrm{mg} / \mathrm{kg})$ & $A I(\%)$ \\
\hline Sham & 10 & - & $2.56 \pm 0.72$ \\
TAC Model & 7 & - & $18.23 \pm 2.47^{\mathrm{a}}$ \\
Fas & 9 & 5 & $15.12 \pm 1.67^{\mathrm{ab}}$ \\
Fas & 10 & 20 & $10.89 \pm 2.92^{\mathrm{ac}}$ \\
Cap & 10 & 50 & $12.34 \pm 2.37^{\mathrm{ac}}$ \\
\hline
\end{tabular}

Note: Compared with the Sham group, ${ }^{a} \mathrm{P}<0.05$; Compared with TAC model group, ${ }^{\mathrm{b}} \mathrm{P}<0.05,{ }^{\mathrm{c}} \mathrm{P}<0.01$ ).

\section{Western Blot Detection of Protein Levels of Myocardial Apoptotic Gene Caspase-3 and Bcl-2}

As displayed in Figure 2,Compared with the control group, significant increases in Caspase-3 protein expression and significant decreases in Blc-2 protein expression in the TAC model group were observed . Furthermore, significantly less Caspase-3 expression and more Blc-2 expression were observed after treatment with Fas (particularly with the $20 \mathrm{mg} / \mathrm{kg}$ dose) or Cap, when compared to the TAC model group.

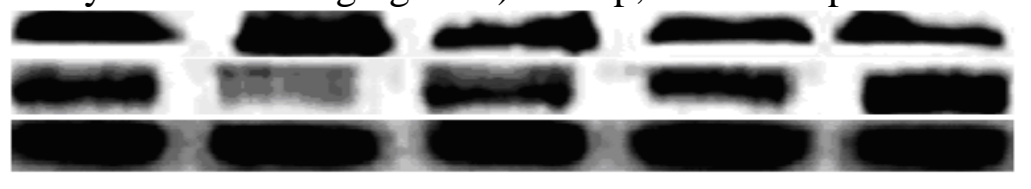

Caspase-3

Bcl-2

$\beta-\operatorname{actin}$

Sham TAC Model Fas $5 \mathrm{mg} / \mathrm{kg} \quad$ Fas $20 \mathrm{mg} / \mathrm{kg} \quad$ Cap50mg/kg

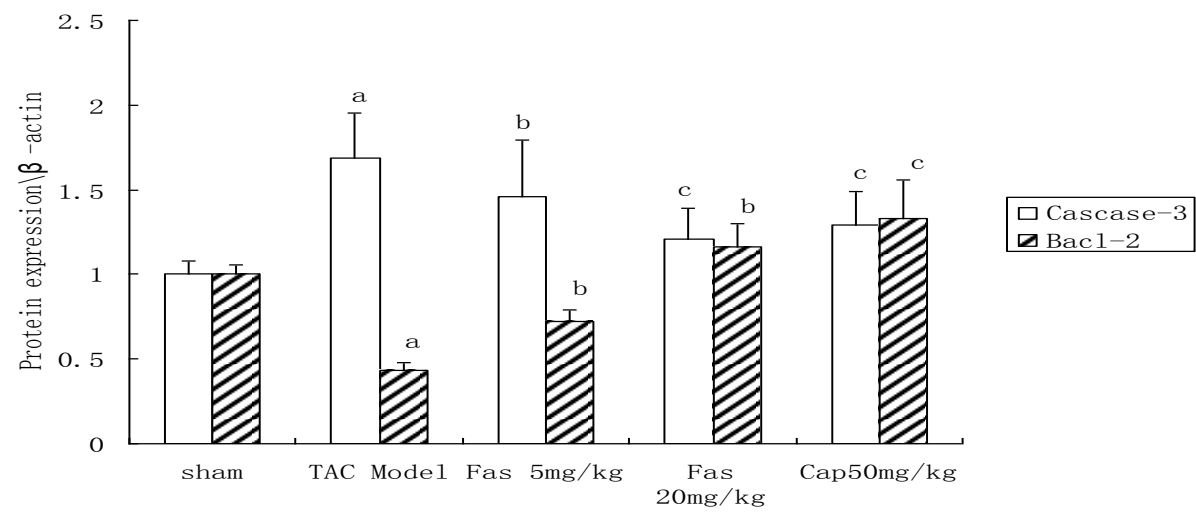

Figure 2. Western blot analysis of myocardial Caspase- 3 and the Bcl-2 protein in each group of rats $(x$ $\pm \mathrm{S}, n=7-10)$; Compared with the Sham group, ${ }^{\mathrm{a}} P<0.05$; Compared with TAC model group, ${ }^{\mathrm{b}} P<0.05,{ }^{\mathrm{c}}$ $P<0.01)$. 


\section{Discussion}

Pressure overloads induced by the TAC rat model can lead to mature cardiac hypertrophy changes. These changes can increase significantly after cardiac after-load, and can cause significant activation of AngII, aldosterone, ET, ANP, and other neurohumoral hormones [12]. These responses are often correlated with clinical myocardial hypertrophy. The typical pathological changes associated with cardiac hypertrophy are usually observed four weeks after surgery $[12,13]$. In this study, the results indicated that rats in the TAC model group showed significant increases in induction of cardiac HWI, LVMI, and myocardial CMD. Rats in this group also presented with increases in blood biochemical parameters, such as AngII, ET, and ANP. These results are in line with the basic characteristics of cardiac hypertrophy, suggesting that this TAC-induced model of cardiac hypertrophy is a successful model for this condition.

The TUNEL technique is a common method of quantitative detection of apoptosis [14]. In recent years, many scholars have reported a major role for apoptosis in the process of cardiac hypertrophy. Proteins involved in apoptosis regulation can be divided into two major categories: pro-apoptotic genes, such as Bax and Fas, and antiapoptotic genes, such as Bcl-2. In particular, the Bcl-2 gene family has been recognized as closely associated with apoptosis [15], as Bcl-2 leads to resistance to myocardial apoptosis. In addition, the caspase family of enzymes is important for activation of apoptosis in mammalian cells. Caspase-3 is considered to be a key protease, as its disintegration promotes apoptosis and wrapped the formation of apoptotic bodies [16]. Studies have shown that cells contain an inactive precursor of Caspase-3 (procaspase-3). When cells are activated with an apoptotic signal, such as a harmful substance or various external stimuli, procaspase- 3 is transformed into active Caspase- 3 by enzymatic cleavage by a specific substrate. the occurrence of DNA involved in apoptosis-dependent protein kinase. Thus, the level of Caspase-3 activation is often used as a reliable indicator of the severity of apoptosis. It is believed that inhibition of Caspase- 3 protein expression may significantly inhibit apoptosis [17]. In this study, we found that the LVMI in TAC-induced rats was significantly higher than that of the control group $(\mathrm{p}<0.05)$, indicating the occurrence of myocardial hypertrophy. The apoptosis rate in the TAC model group was significantly higher than that of the control group $(\mathrm{p}<0.01)$, suggesting that myocardial apoptosis may contribute to the development and progression of cardiac hypertrophy. Myocardial tissues isolated from rats in the Fas or Cap treatment groups also contained apoptotic cells, but AI apoptosis in these groups was significantly lighter than that of the model group after TAC. The results also showed that Fas or Cap treatment reduced Caspase-3 expression and increased Bcl-2 protein expression, compared to TAC model group. We speculate that Fas and Cap might inhibit apoptosis by reducing Caspase-3 protein expression and increasing expression of Bcl2 , which would ultimately reduce cardiac hypertrophy. These observations are consistent with published reports on Cap [18].

The hemodynamics of a whole animal can accurately reflect the pathophysiology of cardiac hypertrophy. Therefore, it is important to observe the effects of drugs on the cardiac hypertrophy model. After myocardial contractility or increased load, LVSP increases. LVEDP represents left ventricular end-diastolic pressure and is a reflection of the important parameters of left ventricular diastolic function. The left ventricular pressure maximum rate of rise and fall $( \pm \mathrm{dP} / \mathrm{dtmax})$ to some extent reflects the rate of change of wall tension. Myocardial function was used to evaluate commonly used indicators. The $+\mathrm{dP} / \mathrm{dtmax}$ to some extent reflects wall tension and 
the rate of change in the contractile function of myocardial fibers. The larger the influence of cardiac preload and after-load, -dp/dtmax reflects left ventricular diastolic function [19]. The experimental results from our study show that, compared with that of the TAC model group, the groups receiving Cap or a high-dose of Fas had significantly $(p<0.01$ or $p<0$ 05) lower LVSP, LVEDP and $\pm d P / d t m a x$ values. Thus, Fas treatment for cardiac hypertrophy in rats showed significant improvement in the blood hemodynamic.

Studies have shown that NO can inhibit cardiac myocytes and vascular proliferation, as well as fibroblast proliferation and synthesis into interstitial collagen. NO thereby inhibits myocardial fibrosis and cardiac remodeling in cardiac hypertrophy and can also antagonize of the vasoconstrictor effect of ET, NO [20, 21], which is considered to be an intrinsic cardiac hypertrophy inhibitory factor, is mainly generated by the activation of guanylate cyclase (GC). GC catalyzes the reaction of guanosine triphosphate to cyclic guanosine monophosphate (3'-5'-cyclic guanosine monophosphate, cGMP), which plays an important role in signal transduction. More specifically, cGMP signaling induces cardiac hypertrophy by suppressing procalcineurin-NFAT signaling pathways that normally inhibit the development of cardiac hypertrophy. AngII neuroendocrine is an important factor in controlling the physiological function of the cardiovascular system, and is also involved in the pathophysiological process of cardiac hypertrophy or heart failure [22]. ET-1 is the strongest known vasoconstrictor and is also a potential cardiac cell growth factor and hypertrophic factor involved in the occurrence of myocardial hypertrophy and development process $[23,24]$. To promote protein synthesis and cardiac hypertrophy, ET-1 and Ang II can activate the same protein kinase $\mathrm{C}$ (PKC) and $\mathrm{Ca}^{2+}$ signal transduction pathways, which are mainly induced in myocardial cells, during fibroblast differentiation..

Oxidative stress is a state of imbalance in the body between production of reactive oxygen species, often generated by normal metabolism, and the body's antioxidant defense system. Oxidative stress is associated with a number of pathologies, including ischemia-reperfusion, atherosclerosis, kidney failure, and endothelial dysfunction, as well as pressure overload-induced left ventricular hypertrophy [25]. Studies have linked production of reactive oxygen species (ROS) with occurrences of cardiac hypertrophy. ROS may affect a variety of cardiac functions and in vivo processes, such as excitation-contraction coupling, gene expression, and a variety of cytokines and growth factors downstream of signal transduction pathways [4]. In TAC-induced guinea pigs, increased endothelial ROS generation reduced left ventricular diastolic function. Thus, it's clear that ROS can cause the occurrence and development of cardiac hypertrophy [26].This study has shown that in Fas intervention groups, levels of AngII, ET, ANP significantly decreased, while NO and SOD levels increased significantly. Fas may inhibit blood AngII, ET, and ANP levels, promote the release of NO, and remove free radicals to prevent cardiac hypertrophy and cardiac development. In recent years, a large number of basic and clinical datum further confirmed that nitric oxide synthase (NOS)plays the cardiovascular role through the synthesis of NO, in the pathological conditions of hypertension, atherosclerosis, heart failure and other diseases, NOS showed dysfunction, NOS uncoupling is an important mechanism which leading to decreased the levels of NO and increased the levels of ROS, correction of NOS uncoupling is expected to become a therapeutic target for cardiovascular disease $[27,28]$. It has been reported that NO can surpress myocardial hypertrophy through inhibiting ROA/ROCK signaling pathways or lower the elevation of ROS levels [29]. So further studies are needed to investigate the precise 
mechanism of Fas on NO .

Many studies have shown that $\mathrm{Ca}^{2+}$ is involved in cardiac hypertrophy. More specifically, $\mathrm{Ca}^{2+}$ overload in myocardial cells and abnormal signal transduction pathways are closely related with the occurrence and development of myocardial hypertrophy [30]. In this study, the results indicated that the resting intracellular free $\mathrm{Ca}^{2+}$ ion concentrations in cardiomyocytes isolated from rats in the TAC group were found that the LVMI increased significantly in the TAC model group, suggesting that overload of intracellular free $\mathrm{Ca}^{2+}$ ion concentrations occurs in cardiac hypertrophy induced by pressure overload of rats. Reduction of this increase was observed following treatment of pressure load-induced rats with either Fas or Cap. In recent years, it is blieved that $\mathrm{Ca}^{2+}$ is a secondary cell messenger that can activate multiple signaling pathways, including the calcium/calmodulin-dependent phosphotase calcineurin/NFAT pathway, the calcium/calmodulin-dependent proteinkinase $(\mathrm{CaMK})$, and the MAP kinases (MAPK) pathway. Among these, the calcineurin/NFAT signaling path-Way appears to play an important role in the development of pathological Cardiac hypertrophy [31, 32].Therefore, the effects of Fas on the intracellular free $\mathrm{Ca}^{2+}$ ion concentrations should be further investigated.

In summary, the results have shown that the methodology of the myocardial hypertrophy model induced by TAC is reliable. Myocardial apoptosis may promote the occurrence and development of cardiac hypertrophy. Fas can improve cardiac function and pathological changes of rats with myocardial hypertrophy. The action of Fas may be related to a reduction in the expression of Caspase- 3 protein, and to an elevation in the expression of Bcl-2 protein. Furthermore, treatment with Fas led to reductions in myocardial apoptosis and blood levels of AngII, ET, and ANP. Fas treatment also promoted NO release, removed free radicals, and reduced the overload of intracellular free $\mathrm{Ca}^{2+}$ ion concentrations in cardiac myocytes. To further clarify the effects of Fas on Caspase-3 and Bcl-2 protein expression, and on changes in intracellular calcium levels in cardiac hypertrophy, it is important to understand the mechanism of action of this drug. This knowledge could provide a theoretical basis for the clinical treatment of myocardial hypertrophy, and therefore is the focus of future studies.

\section{Acknowledgments}

This work was supported by project grants from the Natural Science Foundation of Zhejiang province in China (LY13H310005) and partially supported by project grants from the Key Natural Science Foundation of Zhejiang University of Technology in China (X1168117).

\section{References}

[1] S. Maule, A .Milan, T. Grosso and F. Veglio. Left ventricular hypertrophy in patients with autonomic failure. Am J Hypertens 19:1049(2006).

[2] A.L. Bui, T.B. Horwicha and G.C .Fonarow. Epidemiology and risk profile of heart failure. Nat Rev Cardiol 8: 30(2011).

[3] J. Hou and Y. J. Kang. Regression of pathological cardiac hypertrophy: Signaling pathways and therapeutic targets. Pharmacol Therap 135:337(2012).

[4] A. Rohini, N. Agrawal, C.N. Koyani and R. Singh. Molecular targets and regulators of cardiac hypertrophy. Pharmacol Res 61: 269(2010). 
[5] M.A. Fortuño, S. Ravassa, A. Fortuño, G. Zalba and J. Diez. Cardiomyocyte apoptotic cell death in arterial hypertension: mechanisms and potential management. Hpertension 38:1406(2001).

[6] D.L. Mann, P.M. Barger and D. Burkhoff. Myocardial recovery and the failing heart: myth, magic, or molecular target J Am Coll Cardiol 60: 2465(2012).

[7] Y. Song, H. Cai, N. Gu, C. Qian and S. Cao.. Icariin attenuates cardiac remodelling through down-regulating myocardial apoptosis and matrix metalloproteinase activity in rats with congestive heart failure. J Pharm Pharmacol 63: 541(2011).

[8] A.M. Shah and D.L. Mann. In search of new therapeutic targets and strategies for heart failure: recent advances in basic science. Lancet 38:704(2011).

[9] Z .Hong, Y.J. LI, M .Wang, L.H .Zhang, Z.S. Zhao, F.L. Meng, sis in a rat model of type- 2 diabetes. Acta Pharmacol Sin 32: 999(2011).

[10] D. Georg, J.C., Duerr, S.D. Heinemann, Z.Andreas, L. Beat L .Raissa, R. Wilhelm, M. Fritz, P. Chris, E. Bahman, W. Armin and D. Oliver Myocardial hypertrophy is associated with inflammation and activation of endocannabinoid system in patients with aortic valve stenosis. Life Sci 92:976(2013).

[11] X.Z. Lu , X, H, Yang, J. X. Ye, W. N. Zhong, J. H. Wang, Y. H. Yong, K.J. Chao and Y. J. Huang expression of Calsarcin-1 in myocardial hypertrophy processand function of telmisartan treatment. Bull Chin Pharmacol 289:1253(2012).

[12] R. Huang, L. J. Meng and P.W. Sun. Apoptosis and related gene expression of Caspase-3, Cleaved caspase-3 protein in rat With experimental myocardial ischemia/reperfusion injury. Chin J Crit Care Med 28: 527(2008).

[13] R. A. Lockshin. Programmed cell death: history and future of a concept. J Soc Biol 199: 169(2005).

[14] Q. Zhou Y. B. Xiao, J. Liu, P. Y. Wang, L. Chen, Q. J. Zhong, X. F. Wang. Role of bcl-2 transcriptional regulation induced by calmodulin I in pressure overload rat hypertrophic hearts Acta Physiologica Sinica 57:731(2005). .

[15] A. B. Gustafsson and R.A .Gottlieb. Bcl-2 family members and apoptosis, taken to heart. Am J Physiol Cell Physiol 292: C45(2007).

[16] Q Li , Y.L. Guo, Z .Li and X.Y. Xu he interference of picroside II on the expressions of Caspase-3 and PARP following cerebral ischemia reperfusion injury in rats..Chin Pharmacol Bull 26:342(2010).

[17] L Li. The progress of mechanism of heart hypertrophy. J shenyang Med Coll 11:1(2009).

[18] R. Shenget, Z.L. Gu and M.L. Xie Epigallocatechin gallate, the major component of polyphenols in green tea, inhibits telomere attrition mediated cardiomyocyte apoptosis in cardiac hypertrophy. Inter J Cardiol 162:199(2013).

[19] Y. L. Gai, M. Xu, Y. Bai, H. Xiao, X. W. Ma Z. Z. Lv, J. L. Feng, Y. Y. Zhang and Z. P. Li. Evaluation of left ventricular diastolic dysfunction at the early stage of the pressure overload-induced myocardial remodeling in rats. Chin J Pathophysiol 27:1665(2011). 
[20] J. Vinten-Johansen Involvement of Neutrophils in the Pathogenesis of lethal myocardial repedusion injury. Cardiovasc Res 61:481(2004).

[21] B.Y. Guo, Y. H. Shi, R Han, D. R and Y. J. Li. Influence of Curcumin on Expression of Extracellular Signal Regulated Kinase in Neonatal Rat Cardiac Myocytes in Vitro Induced by Angiotensin II. Chin J Integra Med Cardio/cerebrovas Dis 8: 306(2010).

[22] U. U. Masami, T. Yoshiyuki, A. Yoshitaka, H. Yasutomo, I. Yumi, E. Takashi and Y. Shigeki Evaluation of the renin-angiotensin system in cardiac tissues of cats with pressure-overload cardiac hypertrophy. Am J Vet Res 69:343(2008).

[23] S.M. Motte and R..NaeiEndothelin and antagonists. Pharmacol Ther 110:386(2006).

[24] N .Nian, L.Paul and L. Peter. Inflammatory cytokines and Post myocardial infaretion rernodeling. Cire Res 94: 1543(2004)..

[25] F.Y. Zhong, L.D. Li, Z.H. Huang, L.J. Xiong and J .Ceng..Effects of paniculta lactone on myocardial hypertrophy and antioxidant role in rats. Lishizhen Med Materia Med Res 21: 226(2010).

[26] M. Seddon, Y.H. Looi and A.M. Shah. Oxidative stress and redox signalling in cardiac hypertrophy and heart failure. Heart 93:903(2007).

[27] P. Finckenber and E. Mervaala. Novel regulators and drug targets of cardiac hypertrophy. J Hypertens 18: S33(2010).

[28] B.C. Bernardo, K.L. Weeks, L. Pretorius, J.R. Mcmullen. Molecular distinction between physiological and pathological cardiac hypertrophy :Experimental findings and therapeutic strategies. Pharmacology \& Therapeutics 128:121(2010).

[29] M. Dong, W .Ding, Y .Liao, Y .Liu, D .Yan, Y .Zhang, R. Wang, N .Zheng, S .Liu and J. Liu Polydatin prevents hypertrophy in phenylephrine induced neonatal mouse cardiomyocytes and pressure-overload mouse models. Eur J Pharmacol 746:186(2015).

[30] P.H. Sugden. Signaling pathways in cardiac myocyte hypertrophy. Ann Med 32: 611(2001).

[31] J .Heineke and J.D. Molkentin. Regulation of cardiac hypertrophy by intracellular signalling pathways. Nat Rev Mol Cell Biol 7: 589( 2006).

[32] X. Zhou, Q. Zhang, T. Zhao, X. Bai, W. Yuan, Y. Wu, D. Liu, S. Li, J. Ju, S. Chege Gitau, W. Chu, C. Xu and Y .Lu. Cisapride protects against cardiac hypertrophy via inhibiting the up-regulation of calcineurin and NFATc-3. Eur $\mathrm{J}$ Pharmacol 735:202 (2014). 